\title{
ANALYTIC REGULARITY OF CR MAPS INTO SPHERES
}

\author{
NORDine Mir
}

\begin{abstract}
Let $M \subset \mathbb{C}^{N}$ be a connected real-analytic hypersurface and $\mathbb{S}^{2 N^{\prime}-1} \subset$ $\mathbb{C}^{N^{\prime}}$ the unit real sphere, $N^{\prime}>N \geq 2$. Assume that $M$ does not contain any complex-analytic hypersurface of $\mathbb{C}^{N}$ and that there exists at least one strongly pseudoconvex point on $M$. We show that any CR map $f: M \rightarrow \mathbb{S}^{2 N^{\prime}-1}$ of class $\mathcal{C}^{N^{\prime}-N+1}$ extends holomorphically to a neighborhood of $M$ in $\mathbb{C}^{N}$.
\end{abstract}

\section{Introduction}

In this paper we are interested in the analytic regularity of CR mappings from real-analytic hypersurfaces into higher dimensional unit spheres in complex space. While there is a wide literature deciding when CR maps, of a given smoothness, between two real-analytic hypersurfaces in the same complex space must be real-analytic (see e.g. [BN90, Fo93, BER99, Hu01] for complete references up to 1999), very little is known about the analyticity of such maps when the hypersurfaces lie in complex spaces of different dimension. The case of CR maps with target unit spheres, arising e.g. from the embedding problem for pseudoconvex domains into balls (see e.g. [Fo86, EHZ02]), has attracted the attention of many authors. The first regularity result in such a situation was given by Webster [W79] who showed that any CR map of class $\mathcal{C}^{3}$ from a real-analytic strongly pseudoconvex hypersurface in $\mathbb{C}^{N}$ into the unit sphere $\mathbb{S}^{2 N+1} \subset \mathbb{C}^{N+1}$ is real-analytic on a dense open subset of the source hypersurface. Later Forstnerič [Fo89] generalized Webster's result by showing that the same conclusion holds for any CR map of class $\mathcal{C}^{N^{\prime}-N+1}$ with an arbitrary unit sphere $\mathbb{S}^{2 N^{\prime}-1} \subset \mathbb{C}^{N^{\prime}}$ as a target, $N^{\prime}>N \geq 2$ (see also [Hu94]). He also asked in the same paper whether the real-analyticity, or equivalently, the holomorphic extendability of such maps holds at every point. A partial positive answer in codimension one (i.e. for $N^{\prime}-N=1$ ) was given by Baouendi, Huang and Rothschild [BHR96] for the case of CR maps from a real-algebraic hypersurface of D'Angelo finite type (i.e. not containing any positive dimensional complex-analytic subvariety) in $\mathbb{C}^{N}$ into the unit sphere $\mathbb{S}^{2 N+1} \subset \mathbb{C}^{N+1}$. (In [BHR96], the required smoothness for the maps depends on the so-called D'Angelo type of the reference point, which is always greater or equal to two.) In this paper we prove the following theorem,

Received February 24, 2003.

Revised version received March 20, 2003.

2000 Mathematics Subject Classification. 32H02, 32H04, 32V20, 32V30, 32V40. 
which provides a positive answer to Forstnerič's question and at the same time generalizes the quite independent result of Baouendi-Huang-Rothschild.

Theorem 1.1. Let $M \subset \mathbb{C}^{N}$ be a connected real-analytic hypersurface and $\mathbb{S}^{2 N^{\prime}-1} \subset \mathbb{C}^{N^{\prime}}$ the unit sphere, $N^{\prime}>N \geq 2$. Assume that $M$ does not contain any complex-analytic hypersurface of $\mathbb{C}^{N}$ and that there exists at least one strongly pseudoconvex point on $M$. Then any $C R$ map $f: M \rightarrow \mathbb{S}^{2 N^{\prime}-1}$ of class $\mathcal{C}^{N^{\prime}-N+1}$ extends holomorphically to a neighborhood of $M$ in $\mathbb{C}^{N}$.

Observe that if $M$ is a real hypersurface as in Theorem 1.1 and admits a non-constant CR map into a higher dimensional unit sphere, then it has to be pseudoconvex and therefore the set of strongly pseudoconvex points forms a Zariski open subset of $M$ (see Lemmas 2.3 and 2.4). Hence Theorem 1.1 gives the holomorphic extension of $f$ not only near any strongly pseudoconvex point of $M$, but also near all weakly pseudoconvex ones. It is worth noticing that, under the assumptions of Theorem 1.1, the set of weakly pseudoconvex points of $M$ may even contain complex curves. Example 2.11 below provides a simple illustration of such a situation where Theorem 1.1 applies. On the other hand, Theorem 1.1 in conjunction with Lemma 2.3 (ii) below implies the following corollary for real-analytic hypersurfaces of D'Angelo finite type.

Corollary 1.2. Let $M \subset \mathbb{C}^{N}$ be a real-analytic hypersurface which does not contain any nontrivial complex-analytic subvariety and $\mathbb{S}^{2 N^{\prime}-1} \subset \mathbb{C}^{N^{\prime}}$ the unit sphere, $N^{\prime}>N \geq 2$. Then any $C R$ map $f: M \rightarrow \mathbb{S}^{2 N^{\prime}-1}$ of class $\mathcal{C}^{N^{\prime}-N+1}$ extends holomorphically to a neighborhood of $M$ in $\mathbb{C}^{N}$.

Concerning the initial required regularity in Theorem 1.1, we do not know whether the $\mathcal{C}^{N^{\prime}-N+1}$-smoothness assumption is optimal. It is however known (see [L85, G87, Ha90, D90]) that $\mathcal{C}^{0}$-smoothness (and even $\mathcal{C}^{\alpha}$-smoothness, $0<$ $\alpha<1 / 6$; see [S96]) is not enough to guarantee the holomorphic extendability of any map $f$ in Theorem 1.1. On the other hand, when $M$ is a unit sphere too, it follows from the work of Huang [Hu99] that Theorem 1.1 holds with only $\mathcal{C}^{2}$-smoothness assumption on the map $f$ provided $N^{\prime}<2 N-1$ (see also the previous related work in [CS83, Fa90]). We should also mention that for a map $f$ of class $\mathcal{C}^{\infty}$, Theorem 1.1 follows from the work of Pushnikov [Pu90a, Pu90b] (whose proof, however, is incomplete and contains a gap; see [MMZ03]). In the case where $M$ is assumed to be strongly pseudoconvex (at every point) and $f$ is of class $\mathcal{C}^{N^{\prime}-1}$, Theorem 1.1 was announced by Pinchuk in [Pi83] but a proof has not been published.

Our strategy for the proof of Theorem 1.1 is similar to that of the above mentioned result of Baouendi-Huang-Rothschild [BHR96]. The first step is to prove that in the setting of Theorem 1.1, the map $f$ extends meromorphically to a neighborhood of $M$ in $\mathbb{C}^{N}$. The desired holomorphic extension of $f$ will then follow by applying a result due to Chiappari [C91]. The main novelty of this paper consists in the proof of the above mentioned meromorphic extension of $f$ regardless of the codimension $N^{\prime}-N$ (see Proposition 2.1). The ingredients of the proof rely on another meromorphic extension result for a class of CR ratios 
proved in [MMZ02], and an inductive dichotomy (in Lemma 2.8 below) showing that necessarily each component of the map $f$ belongs to this class of ratios (see also [M02] for a related argument in the one-codimensional case in another context).

\section{Proof of Theorem 1.1}

For basic concepts and notions about CR maps, we refer the reader to [B91, BER99]. Theorem 1.1 is an immediate consequence of the following two results and Lemma 2.3 (i).

Proposition 2.1. Let $M \subset \mathbb{C}^{N}$ and $\mathbb{S}^{2 N^{\prime}-1} \subset \mathbb{C}^{N^{\prime}}$ be as in Theorem 1.1 and $f: M \rightarrow \mathbb{S}^{2 N^{\prime}-1}$ a $C R$ map of class $\mathcal{C}^{N^{\prime}-N+1}$. Then $f$ extends meromorphically to a neighborhood of $M$ in $\mathbb{C}^{N}$.

Theorem 2.2. Let $D \subset \mathbb{C}^{N}$ be a domain, $M \subset \partial D$ a real-analytic hypersurface and $F: D \rightarrow \mathbb{C}^{N^{\prime}}$ a holomorphic map extending continuously up to $M, N^{\prime} \geq$ $N \geq 2$. Assume that $F$ maps $D$ into the unit ball $\mathbb{B}^{2 N^{\prime}-1} \subset \mathbb{C}^{N^{\prime}}$, that $\left.F\right|_{M}$ sends $M$ into the unit sphere $\mathbb{S}^{2 N^{\prime}-1} \subset \mathbb{C}^{N^{\prime}}$ and that $\left.F\right|_{M}$ extends meromorphically to a neighborhood of $M$ in $\mathbb{C}^{N}$. Then $\left.F\right|_{M}$ extends holomorphically to a neighborhood of $M$ in $\mathbb{C}^{N}$.

The remainder of the paper is devoted to the proof of Proposition 2.1 while the second ingredient (Theorem 2.2) was already proved by Chiappari in [C91] (see also [CS90] for a previous version where the source manifold is a sphere). We should also mention that in the case $N^{\prime}=N+1$ and $M$ is a real-algebraic hypersurface of D'Angelo finite type, a result analogous to Proposition 2.1 has been proved in [BHR96].

2.1. Some preliminary facts. We start by collecting a few facts from the known literature. Recall here that a real-analytic hypersurface $M \subset \mathbb{C}^{N}$ is said to be minimal at a point $p_{0} \in M$ if there is no complex-analytic hypersurface of $\mathbb{C}^{N}$ contained in $M$ through $p_{0}$ (see e.g. [Tr86, Tu88, BER99]). The following lemma follows from the same arguments as those of [BHR96, Lemma 6.2].

Lemma 2.3. Let $M \subset \mathbb{C}^{N}$ be a real-analytic hypersurface, $\mathbb{S}^{2 N^{\prime}-1} \subset \mathbb{C}^{N^{\prime}}$ the unit real sphere, $N^{\prime}>N \geq 2$, and let $f: M \rightarrow \mathbb{S}^{2 N^{\prime}-1}$ be a non-constant $C R$ map of class $\mathcal{C}^{2}$. Then,

(i) if $M$ is minimal at every point, it is pseudoconvex and moreover, for every $p \in M, f$ extends near $p$ to a holomorphic map $F$ on the pseudoconvex side of $M$ and $F$ maps this side into the unit ball $\mathbb{B}^{2 N^{\prime}-1}$ of $\mathbb{C}^{N^{\prime}}$.

(ii) if $M$ does not contain any nontrivial complex-analytic subvariety, it is pseudoconvex and the set of strongly pseudoconvex points of $M$ is dense in $M$.

Lemma 2.3 (i) together with an elementary unique continuation argument implies: 
Lemma 2.4. In the situation of Proposition 2.1, if $f$ is not constant, the set of strongly pseudoconvex points of $M$ is dense in $M$.

Lemma 2.4 in conjunction with standard Hopf lemma type arguments (see e.g. [Fo89, Hu94, BHR96, EL02]) implies in particular:

Lemma 2.5. In the situation of Proposition 2.1, if $f$ is not constant, it is an immersion at a generic point of $M$, i.e. there is a dense open subset $\Omega$ of $M$ such that for all $p \in \Omega$ the differential $d f(p): \mathbb{C} T_{p} M \rightarrow \mathbb{C} T_{f(p)} \mathbb{S}^{2 N^{\prime}-1}$ is injective. (Here $\mathbb{C} T_{p} M$ (resp. $\left.\mathbb{C} T_{f(p)} \mathbb{S}^{2 N^{\prime}-1}\right)$ denotes the complexified tangent space of $M$ (resp. of $\left.\mathbb{S}^{2 N^{\prime}-1}\right)$ at $p($ resp. at $f(p))$.)

Finally we state the hypersurface version of a meromorphic extension result proved in [MMZ02, Thereom 2.6] that will be very useful for the proof of Proposition 2.1 .

Theorem 2.6. Let $W \subset \mathbb{C}^{N}, V \subset \mathbb{C}^{k}$ be open subsets, $M \subset W$ a connected realanalytic hypersurface, $G: M \rightarrow V$ a continuous $C R$ map and $\Phi, \Psi: V^{*} \times W \rightarrow \mathbb{C}$ holomorphic functions, where $V^{*}:=\{\bar{\zeta}: \zeta \in V\}$. Assume that $M$ is minimal at every point and that there exists a nonempty open subset of $M$ where $\Psi(\overline{G(z)}, z)$ does not vanish and where the quotient

$$
H(z):=\frac{\Phi(\overline{G(z)}, z)}{\Psi(\overline{G(z)}, z)}
$$

is $C R$. Then $\Psi(\overline{G(z)}, z)$ does not vanish on a dense open subset $\widetilde{M} \subset M$ and $H$ extends from $\widetilde{M}$ meromorphically to a neighborhood of $M$ in $\mathbb{C}^{N}$.

We should mention that a preliminary version of Theorem 2.6, namely the case where $G$ is $\mathcal{C}^{\infty}$ over $M$ and $H$ is $\mathrm{CR}$ on a dense open subset of $M$, is contained in [CPS99]. It will be however important for the proof of Proposition 2.1 (and therefore of Theorem 1.1) to have the continuous version provided by Theorem 2.6.

2.2. Reflection identities and a linear system with coefficients of meromorphic type. We start here the effective proof of Proposition 2.1. It is enough to prove that $f$ extends meromorphically to a neighborhood of a given point $p_{0} \in M$. Without loss of generality we may assume that $p_{0}=f\left(p_{0}\right)=0$, that $f$ is not constant and moreover, that $\mathbb{S}^{2 N^{\prime}-1}$ is replaced by the Heisenberg hypersurface i.e.

$$
\mathbb{H}^{2 N^{\prime}-1}=\left\{\left(z^{\prime}, w^{\prime}\right) \in \mathbb{C}^{N^{\prime}-1} \times \mathbb{C}: \rho^{\prime}\left(z^{\prime}, w^{\prime}, \overline{z^{\prime}}, \overline{w^{\prime}}\right):=\operatorname{Im} w^{\prime}-\left|z^{\prime}\right|^{2}=0\right\},
$$

with $\left|z^{\prime}\right|^{2}=\sum_{j=1}^{N^{\prime}-1}\left|z_{j}^{\prime}\right|^{2}, z^{\prime}=\left(z_{1}^{\prime}, \ldots, z_{N^{\prime}-1}^{\prime}\right)$. Shrinking $M$ if necessary near the origin, we may assume that $M$ is connected and may also choose an open connected neighborhood $U \subset \mathbb{C}^{N}$ of the origin such that $M \subset U$ and such that we are given a family $\mathbb{L}=\left(\bar{L}_{1}, \ldots, \bar{L}_{N-1}\right)$ of $(0,1)$ vector fields with real-analytic 
coefficients in $U$ with $\left.\bar{L}_{1}\right|_{M}, \ldots,\left.\bar{L}_{N-1}\right|_{M}$ spanning $T^{0,1} M$, the $(0,1)$ tangent bundle of $M$. In what follows, for $\gamma=\left(\gamma_{1}, \ldots, \gamma_{N-1}\right) \in \mathbb{N}^{N-1}, \gamma_{1}+\ldots+\gamma_{N-1}=$ : $|\gamma| \leq N^{\prime}-N+1, \bar{L}^{\gamma}$ denotes the standard differential operator $\bar{L}_{1}^{\gamma_{1}} \ldots \bar{L}_{N-1}^{\gamma_{N-1}}$ acting on $\mathcal{C}^{N^{\prime}-N+1}$-smooth functions (or maps) on $M$. It will be convenient to define the following classes of functions over $M$.

Definition 2.7. For $M, f, \mathbb{L}$ as above and $0 \leq l \leq N^{\prime}-N+1$, we let $\mathcal{S}_{l}$ denote the set of functions on $M$ that can be written as a polynomial in $\left(\bar{L}^{\gamma} \bar{f}\right)_{|\gamma| \leq l}$ with coefficients that are real-analytic functions over $M$. Denote also by $\mathcal{S}_{-1}$ the set of all real-analytic functions over $M$. For $-1 \leq l \leq N^{\prime}-N+1$, we also define $\mathcal{R}_{l}$ to be the set of all ratios of the form $a / b$ with $(a, b) \in\left(\mathcal{S}_{l}\right)^{2}$ and $b \not \equiv 0$.

Obviously the class $\mathcal{S}_{l}$ forms a subring of those functions over $M$ that are of class $\mathcal{C}^{N^{\prime}-N+1-l}$ and satisfies $\mathcal{S}_{l} \subset \mathcal{S}_{l+1}$. Note also that since $M$ is everywhere minimal, a function in $\mathcal{S}_{l}$ can not vanish on any open subset of $M$ unless it is identically zero (see e.g. [MMZ02, Section 6]). Therefore any ratio in $\mathcal{R}_{l}$ is defined on a dense open subset of $M$, and can not vanish on any open subset of this dense set unless it is identically zero too. This fact will be useful for the proof of Lemma 2.8 below.

The main observation of this paper relies on the following lemma.

Lemma 2.8. In the situation of Proposition 2.1 and with the above notation, shrinking $M$ around the origin if necessary, each component of the map $f$ can be written as a ratio belonging to $\mathcal{R}_{N^{\prime}-N+1}$.

Proof. In the $\left(z^{\prime}, w^{\prime}\right)$ coordinates we split the map $f$ as follows $f=(\tilde{f}, g)=$ $\left(\widetilde{f}_{1}, \ldots, \widetilde{f}_{N^{\prime}-1}, g\right) \in \mathbb{C}^{N^{\prime}-1} \times \mathbb{C}$. Since $f(M) \subset \mathbb{H}^{2 N^{\prime}-1}$, we have the relation satisfied on $M$

$$
g-\bar{g}=2 i\left(\sum_{\nu=1}^{N^{\prime}-1}\left|\widetilde{f}_{\nu}\right|^{2}\right)
$$

By Lemma 2.5 the map $f$ is an immersion at a generic point and in particular, for a generic point $p \in M, d f(p): T_{p}^{0,1} M \rightarrow T_{f(p)}^{0,1} \mathbb{H}^{2 N^{\prime}-1}$ is injective. Pick such a point $p_{0} \in M$. Then we may select $N-1$ components among those of $\widetilde{f}$, say $\widetilde{f}_{1}, \ldots, \widetilde{f}_{N-1}$, such that the matrix $\left(\bar{L}_{j} \overline{\widetilde{f}}_{k}\right)_{1 \leq j, k \leq N-1}$ is invertible at $p_{0}$. The above matrix $\left(\bar{L}_{j} \overline{\widetilde{f}}_{k}\right)_{1 \leq j, k \leq N-1}$ has necessarily rank $N-1$ in a dense open subset of $M$ since its determinant belongs to the class $\mathcal{S}_{1}$ (see Definition 2.7). Now we split the map $\tilde{f}$ as follows $\widetilde{f}=(h, \psi) \in \mathbb{C}^{N-1} \times \mathbb{C}^{N^{\prime}-N}$ where $h=\left(h_{1}, \ldots, h_{N-1}\right):=\left(\widetilde{f}_{1}, \ldots, \widetilde{f}_{N-1}\right)$ and $\psi=\left(\psi_{1}, \ldots, \psi_{N^{\prime}-N}\right):=$ $\left(\widetilde{f}_{N}, \ldots, \tilde{f}_{N^{\prime}-1}\right)$, so that $f=(h, \psi, g)$. Applying for each $j=1, \ldots, N-1$ the vector field $\bar{L}_{j}$ to $(2.2 .2)$ and using the fact that $f$ is CR, we obtain

$$
-\bar{L}_{j} \bar{g}=2 i\left(\sum_{\nu=1}^{N-1} h_{\nu} \cdot \bar{L}_{j} \bar{h}_{\nu}+\sum_{\nu=1}^{N^{\prime}-N} \psi_{\nu} \cdot \bar{L}_{j} \bar{\psi}_{\nu}\right) \text { on } M \text {. }
$$


Since the $\mathcal{C}^{N^{\prime}-N}$-smooth function given by $M \ni z \mapsto \operatorname{det}\left(\bar{L}_{j} \bar{h}_{\nu}(z)\right)_{1 \leq j, \nu \leq N-1}$ does not vanish on a dense open subset $M_{1}$ of $M$, we may apply Cramer's rule to (2.2.2) and (2.2.3) to obtain a relation of the form

$$
(h, g)=r_{0}^{(1)}+\sum_{\nu=1}^{N^{\prime}-N} \psi_{\nu} \cdot r_{\nu}^{(1)} \quad \text { on } M_{1} .
$$

Here for $\nu=0, \ldots, N^{\prime}-N$, each $r_{\nu}^{(1)}$ is a $\mathbb{C}^{N}$-valued ratio with components in the ring $\mathcal{R}_{1}$, and is defined on $M_{1}$. Since $f$ is of class at least $\mathcal{C}^{2}$, we may again apply for each $j=1, \ldots, N-1$ the vector field $\bar{L}_{j}$ to $(2.2 .4)$ on $M_{1}$ to obtain :

$$
0=\bar{L}_{j} r_{0}^{(1)}+\sum_{\nu=1}^{N^{\prime}-N} \psi_{\nu} \cdot \bar{L}_{j} r_{\nu}^{(1)} \quad \text { on } M_{1} .
$$

Define the following $N(N-1) \times\left(N^{\prime}-N\right)$ matrix with $\mathcal{C}^{N^{\prime}-N-1}$-smooth coefficients over $M_{1}$ by setting

$$
A^{(2)}:=\left(\begin{array}{ccc}
\bar{L}_{1} r_{1}^{(1)} & \ldots & \bar{L}_{1} r_{N^{\prime}-N}^{(1)} \\
\bar{L}_{2} r_{1}^{(1)} & \ldots & \bar{L}_{2} r_{N^{\prime}-N}^{(1)} \\
\vdots & \ldots & \vdots \\
\bar{L}_{N-1} r_{1}^{(1)} & \ldots & \bar{L}_{N-1} r_{N^{\prime}-N}^{(1)}
\end{array}\right),
$$

where each $r_{\nu}^{(1)} \in \mathbb{C}^{N}$ is viewed as a column vector. Since any minor of $A^{(2)}$ has its coefficients in the class $\mathcal{R}_{2}$ the matrix $A^{(2)}$ achieves its maximal rank, that we denote by $n^{(2)}$, on a dense open subset of $M_{1}$. Now similarly to [Fo89, Hu94, BHR96, M02], we come to a dichotomy according to whether $n^{(2)}>0$ or $n^{(2)}=0$.

CASE 1: $n^{(2)}>0$. By taking a suitable minor of the matrix $A^{(2)}$, which does not vanish on a dense open subset $M_{2}$ of $M_{1}$, and using (2.2.5) and Cramer's rule we see that at least one of the components of $\psi$, say $\psi_{N^{\prime}-N}$, can be expressed as an affine combination of the following form:

$$
\psi_{N^{\prime}-N}=c_{0}^{(2)}+\sum_{\nu=1}^{N^{\prime}-N-1} \psi_{\nu} \cdot c_{\nu}^{(2)} .
$$

Here for $\nu=0, \ldots, N^{\prime}-N-1$, each $c_{\nu}^{(2)}$ is a $\mathbb{C}^{N+1}$-valued ratio with components in the ring $\mathcal{R}_{2}$, and is defined on $M_{2}$. By using (2.2.4) and (2.2.6), we may write

$$
\left(h, \psi_{N^{\prime}-N}, g\right)=r_{0}^{(2)}+\sum_{\nu=1}^{N^{\prime}-N-1} \psi_{\nu} \cdot r_{\nu}^{(2)}, \quad \text { on } M_{2},
$$

where each $r_{\nu}^{(2)}$ is a $\mathbb{C}^{N+1}$-valued ratio with components in $\mathcal{R}_{2}$, and is defined on $M_{2}$.

CASE 2: $n^{(2)}=0$. We claim that also in this case, we may reduce the starting system of equations (2.2.4) on $M_{1}$ to another system of the form (2.2.7) that holds on a possibly other dense subset of $M$, possibly after interchanging the 
components of $\psi$ and shrinking $M$ near the origin. For this, note that $n^{(2)}=0$ is equivalent to saying that each ratio $r_{\nu}^{(1)}, \nu=1, \ldots, N^{\prime}-N$, is a CR map on $M_{1}$, and hence $r_{0}^{(1)}$ too in view of (2.2.5). Since each component of each ratio $r_{\nu}^{(1)}$ can clearly be written in the form $(2.1 .1)$ for a suitable $\Psi, \Phi$ and $G$ (see [MMZ02, Lemma 6.1] for more details), we may apply Theorem 2.6 to conclude that each $r_{\nu}^{(1)}$ extends as a meromorphic mapping to a neighborhood of the origin in $\mathbb{C}^{N}$. Let $u_{\nu}$ be a $\mathbb{C}^{N}$-valued holomorphic map and $v_{\nu}$ a nonvanishing holomorphic function both defined near the origin in $\mathbb{C}^{N}$ such that $u_{\nu} / v_{\nu}$ gives the meromorphic extension of $r_{\nu}^{(1)}$ near 0 . Then after shrinking $M$ near the origin if necessary, we may rewrite (2.2.4) as follows

$$
(h(z), g(z))=\frac{u_{0}(z)}{v_{0}(z)}+\sum_{\nu=1}^{N^{\prime}-N} \psi_{\nu}(z) \frac{u_{\nu}(z)}{v_{\nu}(z)}, z \in \widetilde{M}_{1},
$$

on some suitable dense open subset $\widetilde{M}_{1}$ of $M$. Following the splitting used in (2.2.8), for any $\nu=0, \ldots, N^{\prime}-N$, we write $u_{\nu}=\left(u_{\nu}^{\prime}, \hat{u}_{\nu}\right) \in \mathbb{C}^{N-1} \times \mathbb{C}$. Consider the family $\left(P_{z}\right)_{z \in \tilde{M}_{1}}$ of $N^{\prime}-N$ dimensional affine complex planes defined via the following parametrization $\eta_{z}: \mathbb{C}^{N^{\prime}-N} \rightarrow \mathbb{C}^{N^{\prime}}, z \in \widetilde{M}_{1}$,

$$
\begin{aligned}
t= & \left(t_{1}, \ldots, t_{N^{\prime}-N}\right) \mapsto \\
& \left(\frac{u_{0}^{\prime}(z)}{v_{0}(z)}+\sum_{\nu=1}^{N^{\prime}-N} t_{\nu} \frac{u_{\nu}^{\prime}(z)}{v_{\nu}(z)}, t_{1}, \ldots, t_{N^{\prime}-N}, \frac{\hat{u}_{0}(z)}{v_{0}(z)}+\sum_{\nu=1}^{N^{\prime}-N} t_{\nu} \frac{\hat{u}_{\nu}(z)}{v_{\nu}(z)}\right) .
\end{aligned}
$$

Since the Heisenberg hypersurface cannot contain any positive-dimensional affine complex subspace, we have $P_{z} \not \subset \mathbb{H}^{2 N^{\prime}-1}$ for all $z \in \widetilde{M}_{1}$ i.e.

$$
\rho^{\prime}\left(\eta_{z}(t), \overline{\eta_{z}(t)}\right) \not \equiv 0, \text { for } z \in \widetilde{M}_{1}, t \in \mathbb{C}^{N^{\prime}-N},
$$

where $\rho^{\prime}$ is given by $(2.2 .1)$. It is clear that $\rho^{\prime}\left(\eta_{z}(t), \overline{\eta_{z}(t)}\right)$ may be written as follows

$$
\mu+\sum_{i=1}^{N^{\prime}-N} \lambda_{i} \overline{t_{i}}+\sum_{j=1}^{N^{\prime}-N} t_{j}\left(\sum_{k=1}^{N^{\prime}-N} \xi_{j k} \overline{t_{k}}+\sigma_{j k}\right),
$$

where $\mu, \lambda_{i}, \xi_{j k}$ and $\sigma_{j k}$ are ratios in $\mathcal{R}_{-1}$ and defined on $\widetilde{M}_{1}$. (We have deliberately omitted to write the dependence on $z$ for those ratios in (2.2.11).) But since $f$ sends $M$ into $\mathbb{H}^{2 N^{\prime}-1}$, in view of (2.2.9) and (2.2.8), we have $\rho^{\prime}\left(\eta_{z}(\psi(z)), \overline{\eta_{z}(\psi(z))}\right)=0$ for $z \in \widetilde{M}_{1}$ i.e.

$$
\mu+\sum_{i=1}^{N^{\prime}-N} \lambda_{i} \overline{\psi_{i}}+\sum_{j=1}^{N^{\prime}-N} \psi_{j}\left(\sum_{k=1}^{N^{\prime}-N}\left(\xi_{j k} \overline{\psi_{k}}+\sigma_{j k}\right)\right)=0 \text { on } \widetilde{M}_{1}
$$

Here again we come to a dichotomy.

SubCASE 2.1: there exists $j_{0} \in\left\{1, \ldots, N^{\prime}-N\right\}$ such that $\sum_{k=1}^{N^{\prime}-N}\left(\xi_{j_{0} k} \overline{\psi_{k}}+\right.$ $\left.\sigma_{j_{0} k}\right) \not \equiv 0$ (as a ratio in $\mathcal{R}_{0}$ ). Then from $(2.2 .12)$ we see that $\psi_{j_{0}}$ can be written 
as an affine combination, with coefficients in $\mathcal{R}_{0} \subset \mathcal{R}_{2}$, of the other components of $\psi$. Using (2.2.4) we see that the claim mentioned at the very beginning of CASE 2 is proved.

SubCASE 2.2: for any $j \in\left\{1, \ldots, N^{\prime}-N\right\}$,

$$
\sum_{k=1}^{N^{\prime}-N}\left(\xi_{j k} \overline{\psi_{k}}+\sigma_{j k}\right) \equiv 0 \text {, on } \widetilde{M}_{1} \text {. }
$$

If there exists $j_{1}, k_{1} \in\left\{1, \ldots, N^{\prime}-N\right\}$ such that $\xi_{k_{1} j_{1}} \not \equiv 0$, then after taking complex conjugates of $(2.2 .13), \psi_{k_{1}}$ can be written as an affine combination, with coefficients in $\mathcal{R}_{-1} \subset \mathcal{R}_{2}$, of the other components of $\psi$. The desired claim therefore follows as in Subcase 2.1. If not, then necessarily all $\xi_{j k}$ and $\sum_{1 \leq k \leq N^{\prime}-N} \sigma_{j k}$ (by $(2.2 .13)$ ) are identically zero. Equation (2.2.12) therefore leads to $\mu+\sum_{1 \leq i \leq N^{\prime}-N} \lambda_{i} \overline{\psi_{i}}=0$. Then there exists necessarily $i_{2} \in\left\{1, \ldots, N^{\prime}-N\right\}$ such that $\lambda_{i_{2}} \not \equiv 0$ since otherwise all quantities $\mu, \lambda_{i}, \xi_{j k}$ and $\sum_{1 \leq k \leq N^{\prime}-N} \sigma_{j k}$ would be identically zero, which would contradict (2.2.10) in view of $(2.2 .11)$. Hence here again we obtain that $\psi_{i_{2}}$ can be written as an affine combination, with coefficients in $\mathcal{R}_{-1} \subset \mathcal{R}_{2}$, of the other components of $\psi$, which completes, as explained before, the proof of the claim announced at the beginning of CASE 2 .

We are now able to finish the proof of Lemma 2.8. The conclusion of the dichotomy studied in CASES 1 and 2 states that we can always reduce a system of the form (2.2.4) with coefficients in $\mathcal{R}_{1}$ to another system of the form (2.2.7) with coefficients in $\mathcal{R}_{2}$. If $N^{\prime}=N+1$, we are clearly done. If not, by using similar arguments as in the above process, it is easy to show that the obtained system with coefficients in $\mathcal{R}_{2}$ can also be reduced to a system where $N+2$ components of the map can be expressed as an affine combination of the other ones with coefficients in $\mathcal{R}_{3}$. (Note that the only ingredients needed for such a process are Theorem 2.6 and the fact that the Heisenberg hypersurface does not contain any complex affine subspace of positive dimension.) By pursuing this procedure $N^{\prime}-N-2$ more times, we conclude that each component of $f$ can be written as a ratio belonging to $\mathcal{R}_{N^{\prime}-N+1}$. The proof of Lemma 2.8 is complete.

2.3. Completion of the proof of Proposition 2.1. By Lemma 2.8 we know that after shrinking $M$ near the origin, each component of $f$ agrees on a dense open subset of $M$ with a ratio in the class $\mathcal{R}_{N^{\prime}-N+1}$. Moreover since $M$ is minimal at $0, f$ extends to a holomorphic map $F$ defined on one side of $M$ near the origin [BT84, Tr86]. Therefore by using this extension $F$, it is easy to see that any ratio in the class $\mathcal{R}_{N^{\prime}-N+1}$ can be written in the form (2.1.1) for a suitable $\Psi, \Phi$ and $G$ (for further details on that matter see [MMZ02, Lemma 6.1]). Since each component of $f$ is CR, we conclude from Theorem 2.6 that each such component extends meromorphically to a neighborhood of the origin in $\mathbb{C}^{N}$. This completes the proof of Proposition 2.1 and hence that of Theorem 1.1. 
Remark 2.9. In the proof of Theorem 1.1, the assumption that $M$ does not contain any complex-analytic hypersurface of $\mathbb{C}^{N}$ is essential when applying several times Theorem 2.6. We do not know whether Theorem 1.1 holds without such an assumption. (The main problem without such a hypothesis would be to prove the meromorphic extension of the map $f$ near any non-minimal point.) On the other hand, the assumption that $M$ be strongly pseudoconvex at some point in Theorem 1.1 is used to know that any non-constant CR map $f: M \rightarrow \mathbb{S}^{2 N^{\prime}-1}$ is a local embedding near a generic point of $M$. Without assuming the existence of a strongly pseudoconvex point on $M$, the conclusion of Theorem 1.1 still holds if $f$ is assumed to be of class $\mathcal{C}^{N^{\prime}-r_{f}+1}$ where $r_{f}:=\operatorname{Sup}\left\{\left.\operatorname{Rank} d f(p)\right|_{T_{p}^{0,1} M}: p \in\right.$ $M\}$ (with a trivial modification in the beginning of the proof of Lemma 2.8). This implies in particular that Theorem 1.1 still holds for all CR maps of class $\mathcal{C}^{N^{\prime}}$ without assuming the existence of any strongly pseudoconvex point on $M$. We leave the details to the reader.

We conclude this paper by giving examples of situations covered by Theorem 1.1 and Corollary 1.2 where previously known results do not apply.

Example 2.10. Given nonnegative integers $k, l, k \geq 1, l \geq 3$, and $h$ a holomorphic function defined near the origin in $\mathbb{C}$ with $h(0)=h^{\prime}(0)=0$, define $M_{k} \subset \mathbb{C}^{2}$ to be the real-analytic hypersurface through the origin given by

$$
M_{k}:=\left\{\left(z_{1}, z_{2}\right) \in \mathbb{C}^{2}: \operatorname{Im} z_{2}=\left|z_{1}\right|^{2 k}+\left|h\left(z_{1}\right)\right|^{2}\right\} .
$$

Note that there are non-constant CR maps from $M_{k}$ into the Heisenberg representation

$$
\mathbb{H}^{2 l-1}:=\left\{\left(w_{1}, \ldots, w_{l}\right) \in \mathbb{C}^{l}: \operatorname{Im} w_{l}=\left|w_{1}\right|^{2}+\ldots+\left|w_{l-1}\right|^{2}\right\}
$$

of the unit sphere in $\mathbb{C}^{l}$, namely the restriction to $M_{k}$ of the holomorphic map

$$
\left(z_{1}, z_{2}\right) \mapsto(z_{1}^{k}, h\left(z_{1}\right), \underbrace{0, \ldots, 0}_{l-3 \text { times }}, z_{2}) .
$$

For $k=1, M_{1}$ is strongly pseudoconvex and the analyticity (at every point of $M_{1}$ ) of $\mathcal{C}^{l-1}$-smooth CR maps from $M_{1}$ into $\mathbb{H}^{2 l-1}$ follows from Corollary 1.2 but not from [Fo89]. In the case $l=3$, the regularity of such maps does not follow neither from [BHR96] if the function $h$ is chosen not be algebraic.

For $k>1, M_{k}$ is weakly pseudoconvex of D'Angelo finite type and the set of weakly pseudoconvex points is a real line. Here again the analytic regularity (at every point of $M_{k}$ ) of $\mathcal{C}^{l-1}$-smooth CR maps from $M_{k}$ into $\mathbb{H}^{2 l-1}$ follows from Corollary 1.2 but not from [Fo89]. Moreover, even if $l=3$ and $h$ is chosen to be algebraic, the analyticity at the origin of such maps does not follow from [BHR96] since the type of $M_{k}$ at 0 is clearly greater or equal to 4 (and the required initial order of smoothness for the maps in [BHR96] equals this type).

Example 2.11. Let $M \subset \mathbb{C}^{3}$ be the real-algebraic hypersurface given by $M:=$ $\left\{\left(z_{1}, z_{2}, z_{3}\right) \in \mathbb{C}^{3}: \operatorname{Im} z_{3}=\left|z_{1} z_{2}\right|^{2}+\left|z_{2}\right|^{2}\right\}$. Then $M$ obviously contains a complex line through the origin and the set of strongly pseudoconvex points of $M$ is dense in $M$. (Note also that $M$ is not essentially finite at the origin in the 
sense of [BJT85].) For any nonnegative integer $l \geq 4$, the restriction to $M$ of the following holomorphic map

$$
\left(z_{1}, z_{2}, z_{3}\right) \mapsto(z_{1} z_{2}, z_{2}, \underbrace{0, \ldots, 0}_{l-3 \text { times }}, z_{3}) \in \mathbb{C}^{l}
$$

maps $M$ into $\mathbb{H}^{2 l-1}$ (given by (2.3.1)), and is near a generic point a local embedding. The analyticity (at every point of $M$ ) of $\mathcal{C}^{l-2}$-smooth CR maps from $M$ into $\mathbb{H}^{2 l-1}$ follows from Theorem 1.1 but neither from [Fo89] nor from Corollary 1.2 .

\section{Acknowledgements}

This paper was written while I was visiting UCSD. I would like to thank this institution for its hospitality and M. S. Baouendi, M. Derridj, P. Ebenfelt, B. Lamel, F. Meylan, L. P. Rothschild and D. Zaitsev for useful discussions and comments on this paper. I am also grateful to the referee for several helpful remarks.

\section{References}

[BER99] M. S. Baouendi, P. Ebenfelt, L. P. Rothschild, Real submanifolds in complex space and their mappings, Princeton Math. Series 47, Princeton Univ. Press, 1999.

[BHR96] M. S. Baouendi, X. Huang, L. P. Rothschild, Regularity of CR mappings between algebraic hypersurfaces, Invent. Math. 125 (1996), 13-36.

[BJT85] M.S. Baouendi, H. Jacobowitz, F. Treves, On the analyticity of CR mappings, Ann. of Math. (2) 122 (1985), 365-400.

[BT84] M.S. Baouendi, F. Treves, About the holomorphic extension of CR functions on real hypersurfaces in complex space, Duke Math. J. 51 (1984), 77-107.

[BN90] S. R. Bell, R. Narasimhan, Proper holomorphic mappings of complex spaces, Several complex variables, VI, 1-38, Encyclopaedia Math. Sci., 69, Springer, Berlin, 1990.

[B91] A. Boggess, CR manifolds and the tangential Cauchy-Riemann complex, Studies in Advanced Mathematics. CRC Press, Boca Raton, FL, 1991.

[C91] S. Chiappari, Holomorphic extension of proper meromorphic mappings, Michigan Math. J. 38 (1991), 167-174.

[CS83] J. A. Cima, T. J. Suffridge, A reflection principle with applications to proper holomorphic mappings, Math. Ann. 265 (1983), 489-500.

[CS90] Boundary behavior of rational proper maps, Duke Math. J. 60, (1990), $135-138$.

[CPS99] B. Coupet, S. Pinchuk, A. Sukhov, Analyticité des applications CR, C. R. Acad. Sci. Paris Sér. I Math. 329 (1999), 489-494.

[D90] A. Dor, Proper holomorphic maps between balls in one co-dimension, Ark. Mat. 28 (1990), 49-100.

[EHZ02] P. Ebenfelt, X. Huang, D. Zaitsev, The equivalence problem and rigidity for hypersurfaces embedded into hyperquadrics, preprint, 2002:

http://www.arxiv.org/abs/math.CV/0211024

[EL02] P. Ebenfelt, B. Lamel, Finite jet determination of constantly degenerate CR embeddings, preprint, 2002: http://www.arxiv.org/abs/math.CV/0206287

[Fa90] J. Faran, A reflection principle for proper holomorphic mappings and geometric invariants, Math. Z. 203 (1990), 363-377.

[Fo86] F. Forstnerič, Embedding strictly pseudoconvex domains into balls, Trans. Amer. Math. Soc. 295 (1986), 347-368. 
[Fo89] Extending proper holomorphic mappings of positive codimension, Invent. Math. 95, (1989), 31-61.

[Fo93] $\quad$ Proper holomorphic mappings: a survey, Several complex variables (Stockholm, 1987/1988), 297-363, Math. Notes, 38, Princeton Univ. Press, Princeton, NJ, 1993.

[G87] J. Globevnik, Boundary interpolation by proper holomorphic maps, Math. Z. 194 (1987), 365-373.

[Ha90] M. Hakim, Applications holomorphes propres continues de domaines strictement pseudoconvexes de $\mathbb{C}^{n}$ dans la boule unité de $\mathbb{C}^{n+1}$, Duke Math. J. 60 (1990), $115-133$.

[Hu94] X. Huang, On the mapping problem for algebraic real hypersurfaces in the complex spaces of different dimensions, Ann. Inst. Fourier (Grenoble) 44 (1994), 433-463.

[Hu99]_ On a linearity problem for proper holomorphic maps between balls in complex spaces of different dimensions, J. Differential Geom. 51 (1999), 13-33.

[Hu01] _ On some problems in several complex variables and CR geometry, First International Congress of Chinese Mathematicians (Beijing, 1998), 383-396, AMS/IP Stud. Adv. Math., 20, Amer. Math. Soc., Providence, RI, 2001.

[L85] E. Low, Embeddings and proper holomorphic maps of strictly pseudoconvex domains into polydiscs and balls, Math. Z. 190 (1985), 401-410.

[MMZ02] F. Meylan, N. Mir, D. Zaitsev, Analytic regularity of CR-mappings, Math. Res. Lett. 9 (2002), 71-91.

[MMZ03] Holomorphic extension of smooth CR-mappings between real-analytic and real-algebraic CR-manifolds, Asian J. Math. (2003), to appear in the Special issue dedicated to Y.-T. Siu on the occasion of his sixty birthday.

[M02] N. Mir, Convergence of formal embeddings between real-analytic hypersurfaces in codimension one, J. Differential Geom. 62 (2002), 163-173.

[Pi83] S.I. Pinchuk, Analytic continuation of holomorphic mappings and problems of holomorphic classification of multi-dimensional domains, (Russian) Mat. Zametki 33 (1983), 301-314. English translation in Math. Notes 33 (1983), 151-157.

[Pu90a] A. Y. Pushnikov, Holomorphicity of CR-mappings into a space of large dimension, Mat. Zametki 48 (1990), 147-149.

[Pu90b] On the holomorphy of CR-mappings of real analytic hypersurfaces, Complex analysis and differential equations (Russian), 76-84, Bashkir. Gos. Univ., Ufa, 1990.

[S96] B. Stensones, Proper maps which are Lipschitz $\alpha$ up to the boundary, J. Geom. Anal. 6 (1996), 317-339.

[Tr86] J.-M. Trépreau, Sur le prolongement holomorphe des fonctions CR définies sur une hypersurface réelle de classe $C^{2}$ dans $\mathbb{C}^{n}$, Invent. Math. 83 (1986), 583-592.

[Tu88] A. E. Tumanov, Extension of CR-functions into a wedge from a manifold of finite type, (Russian). Mat. Sb. (N.S.) 136 (178) (1988), 128-139. English translation in Math. USSR-Sb. 64 (1989), 129-140.

[W79] S. M. Webster, On mapping an $n$-ball into an $n+1$-ball in complex space, Pacific J. Math. 81 (1979), 267-272.

Université de Rouen, Laboratoire de Mathématiques Raphä̈l Salem, UMR 6085 CNRS, 76821 Mont-Saint-Aignan Cedex, France.

E-mail address: Nordine.Mir@univ-rouen.fr 